\title{
Star Cluster Disruption by a Supermassive Black Hole Binary
}

\author{
Elisa Bortolas* \\ INAF, Osservatorio Astronomico di Padova, Vicolo dell'Osservatorio 5, I-35122 Padova, Italy \\ Dipartimento di Fisica e Astronomia 'Galileo Galilei', Università di Padova, Vicolo \\ dell'Osservatorio 3, I-35122 Padova, Italy \\ E-mail: elisa.bortolas@phd.unipd.it
}

\section{Michela Mapelli}

INAF, Osservatorio Astronomico di Padova, vicolo dell'Osservatorio 5, I-35122 Padova, Italy Institute for Astrophysics and Particle Physics, University of Innsbruck, Technikerstraße 25/8, A-6020 Innsbruck, Austria

INFN, Milano Bicocca, Piazza della Scienza 3, I-20126 Milano, Italy

E-mail: michela.mapelli@oapd.inaf.it

\section{Mario Spera}

INAF, Osservatorio Astronomico di Padova, vicolo dell'Osservatorio 5, I-35122 Padova, Italy Institute for Astrophysics and Particle Physics, University of Innsbruck, Technikerstraße 25/8, A-6020 Innsbruck, Austria INFN, Milano Bicocca, Piazza della Scienza 3, I-20126 Milano, Italy

E-mail: mario.spera@uibk.ac.at

Binary supermassive black holes (BBHs) are expected to be one of the most powerful sources of low-frequency gravitational waves (GWs) for future space-borne detectors. Prior to the GW emission stage, BBHs evolving in gas-poor nuclei shrink primarily through the slingshot ejection of stars approaching the $\mathrm{BBH}$ from sufficiently close distances. Here we address the possibility that the $\mathrm{BBH}$ shrinking rate is enhanced through the infall of a star cluster (SC) onto the BBH. We present the results of direct summation $N$-body simulations exploring different orbits for the $\mathrm{SC}$ infall, and we show that SCs reaching the BBH on non-zero angular momentum orbits (with eccentricity 0.75 ) fail to enhance the $\mathrm{BBH}$ hardening, while SCs approaching the $\mathrm{BBH}$ on radial orbits reduce the $\mathrm{BBH}$ separation by $\sim 10 \%$ in less than $10 \mathrm{Myr}$, effectively shortening the $\mathrm{BBH}$ path towards GWs.

GRAvitational-waves Science\& technology Symposium - GRASS2018

1-2 March 2018

Palazzo Moroni, Padova (Italy)

\footnotetext{
*Speaker.
} 


\section{Introduction}

Binary supermassive black holes (BBHs) are a natural by-product of galaxy mergers; as such, they are expected to form in large numbers along cosmic time [1]. The study of BBHs has received considerable attention in the last decades: when the $\mathrm{BBH}$ members reach separations of the order of a few mpc, they coalesce into a single supermassive black hole (SMBH) via a burst of lowfrequency gravitational waves (GWs) [2]. Such GW sources will shine in the band of the future space-borne LISA observatory, making BBH mergers among the main targets for the LISA mission [3].

In the early stages of a galaxy merger, dynamical friction drives the SMBHs toward the centre of their common potential well. When the BBH separation drops below parsec scales, the BBH shrinking in gas-poor nuclei is primarily driven by three body scatterings ('slingshot ejections') of stars on sufficiently low angular momentum orbits [1]. At the beginning of the slingshot phase, the BBH promptly expels from the galaxy almost all stars able to reach its neighbourhood. After that, the BBH shrinking can slow down considerably and even stop at roughly parsec scale, unless any physical processes can guarantee a steady repopulation of the binary loss cone (i.e. the region of phase-space containing stars with sufficiently low angular momentum) [4]. For this reason, a number of studies in the last decades have investigated whether BBHs can efficiently merge in gaspoor environments [1,5]. Currently, a general solution to the 'final parsec problem' is believed to reside in the non-sphericity of the host galaxy [6, 7, and references therein]. If the host galaxy is triaxial, gravitational torques ensure a steady scattering of stars into the BBH loss cone [8]; given that galaxy mergers are expected to naturally induce non-sphericity in the merger relic [9], most BBHs are believed to find their way to coalescence within a few Gyr [6].

Here we propose a novel possible way to shorten the BBH path to coalescence, via the infall of a massive stellar cluster (SC) onto a parsec-scale BBH. In fact, young SCs are common in galactic nuclei, and may form in a burst of star formation following the galaxy merger [10]; then, SCs may sink towards the centre of the system via dynamical friction, interacting with the $\mathrm{BBH}$, and possibly significantly contributing to its hardening. The work presented here is a summary of [11]; a further study addressing the effect of SC infalls onto BBHs can be found at [12].

\section{Methods and initial conditions}

We simulate the infall of a SC onto a BBH adopting the highly accurate, direct summation $N$-body code HiGPUs [13]. We place two $10^{6} \mathrm{M}_{\odot} \mathrm{SMBHs}$ on a circular orbit with semimajor axis $a=1 \mathrm{pc}$; the BBH centre of mass coincides with the bottom of the galactic potential well, modelled as a rigid Dehnen profile [14] with total mass $5 \times 10^{10} \mathrm{M}_{\odot}$, scale radius $250 \mathrm{pc}$, and inner density slope $\gamma=0.5$. $\mathrm{A} \approx 8 \times 10^{4} \mathrm{M}_{\odot}$ SC is set at $20 \mathrm{pc}$ from the $\mathrm{BBH}$ centre of mass; the SC follows an isotropic King density profile [15] with core radius $r_{k}=0.4 \mathrm{pc}$, and the SC mass spectrum is modelled with a Kroupa mass function [16]. We explore three different configurations for the cluster infall: in run $1 \mathrm{p}(2 \mathrm{p})$, the SC is initially at rest, and its orbital plane is perpendicular (coplanar) with respect to the $\mathrm{BBH}$ orbital plane; in run $3 \mathrm{p}$, the $\mathrm{SC}$ is placed at the apoapsis of an eccentric orbit with eccentricity 0.75 , coplanar with the $\mathrm{BBH}$, with counter-aligned angular momentum. Each simulation is integrated for $10 \mathrm{Myr}$. 


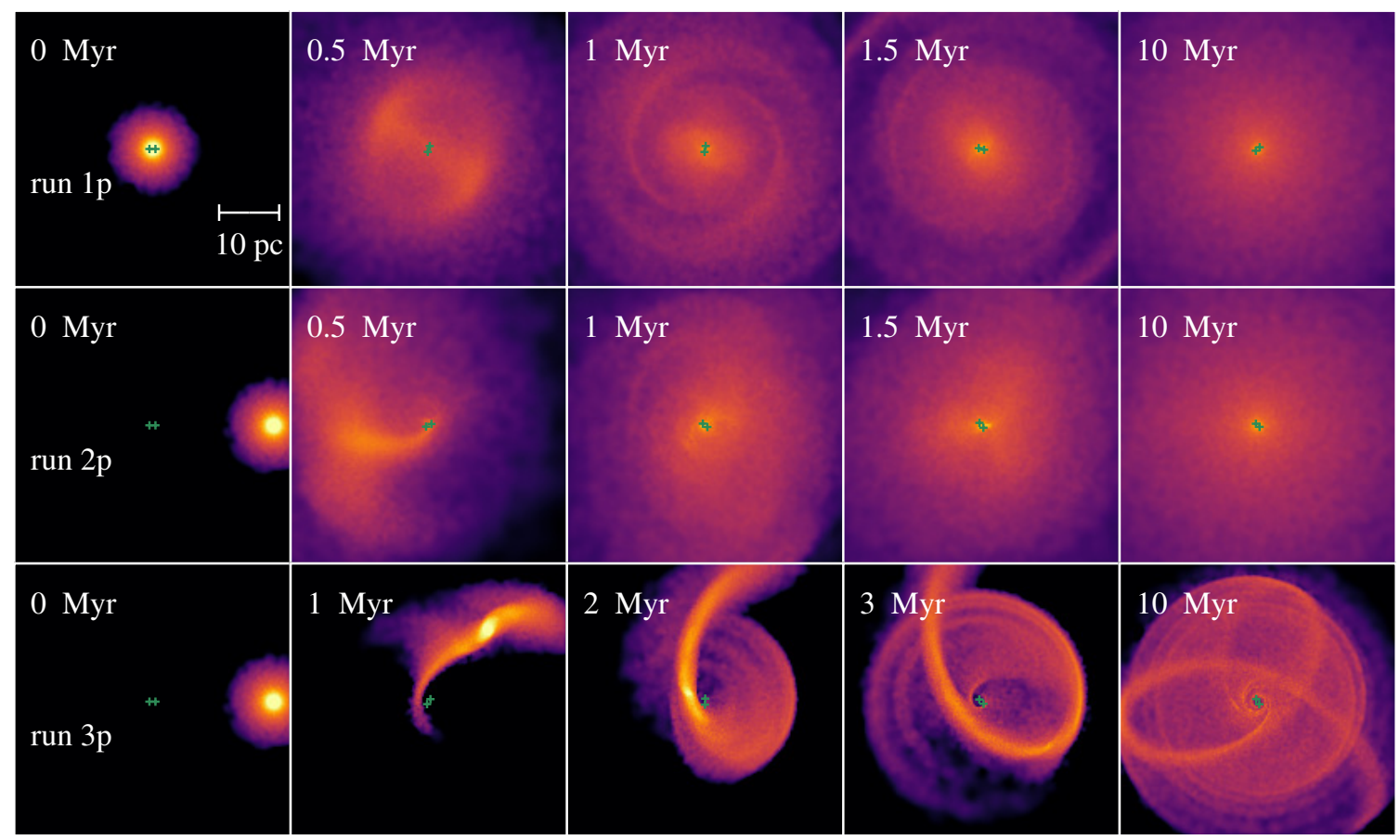

Figure 1: Time evolution of the stellar surface density projected on the $\mathrm{BBH}$ orbital plane for runs 1p (top row), $2 p$ (central row), $3 p$ (bottom row). The green central crosses mark the position of the two SMBHs. The colour coding is logarithmic, and it refers to the smoothed projected mass density of stars, ranging from $10^{-1}$ (black) to $10^{4} \mathrm{M}_{\odot} \mathrm{pc}^{-2}$ (white).

\section{Results}

Fig. 1 shows different snapshots of the simulations projected on the $\mathrm{BBH}$ orbital plane. The $\mathrm{BBH}$ response to the SC infall significantly depends on the SC orbital angular momentum.

\subsection{Radially infalling SCs}

If the $\mathrm{SC}$ is in free fall (i.e. it has zero initial angular momentum, as in runs $1 \mathrm{p}$ and $2 \mathrm{p}$ ), its interaction with the $\mathrm{BBH}$ is rather violent: the cluster gets completely destroyed within the first $1-2$ Myr. After roughly $10 \mathrm{Myr}$, the SC stars are isotropically distributed around the $\mathrm{BBH}$, and the density profile of the cluster remnant, centred on the BBH centre of mass, falls off as $\rho(r) \propto r^{-2}$ between $\approx 1$ and $20 \mathrm{pc}$.

The time evolution of the BBH separation and semimajor axis is shown in the left-hand panel of Fig. 2: after $10 \mathrm{Myr}$, the BBH has shrunk by $\approx 10(13)$ per cent in run $1 \mathrm{p}(2 \mathrm{p})^{1}$; the $\mathrm{BBH}$ hardening is more efficient when the SC infall is coplanar, as in this configuration the relative velocity between the $\mathrm{SC}$ and the $\mathrm{BBH}$ members is lower. In the radial runs, the SC induced $\mathrm{BBH}$ hardening rate $s=d\left(a^{-1}\right) / d t$ after $3.5 \mathrm{Myr}$ is $\sim 10^{-2} \mathrm{pc}^{-1} \mathrm{Myr}^{-1}$. The BBH eccentricity $e$ is not significantly influenced by the interaction: in fact, $e<0.1$ at all times.

\footnotetext{
${ }^{1}$ The BBH semimajor axis, as shown in the left-hand panel of Fig. 2, initially does not shrink monotonically: this results from the fact that the $\mathrm{BBH}$ is marginally soft with respect to the initial infalling SC.
} 

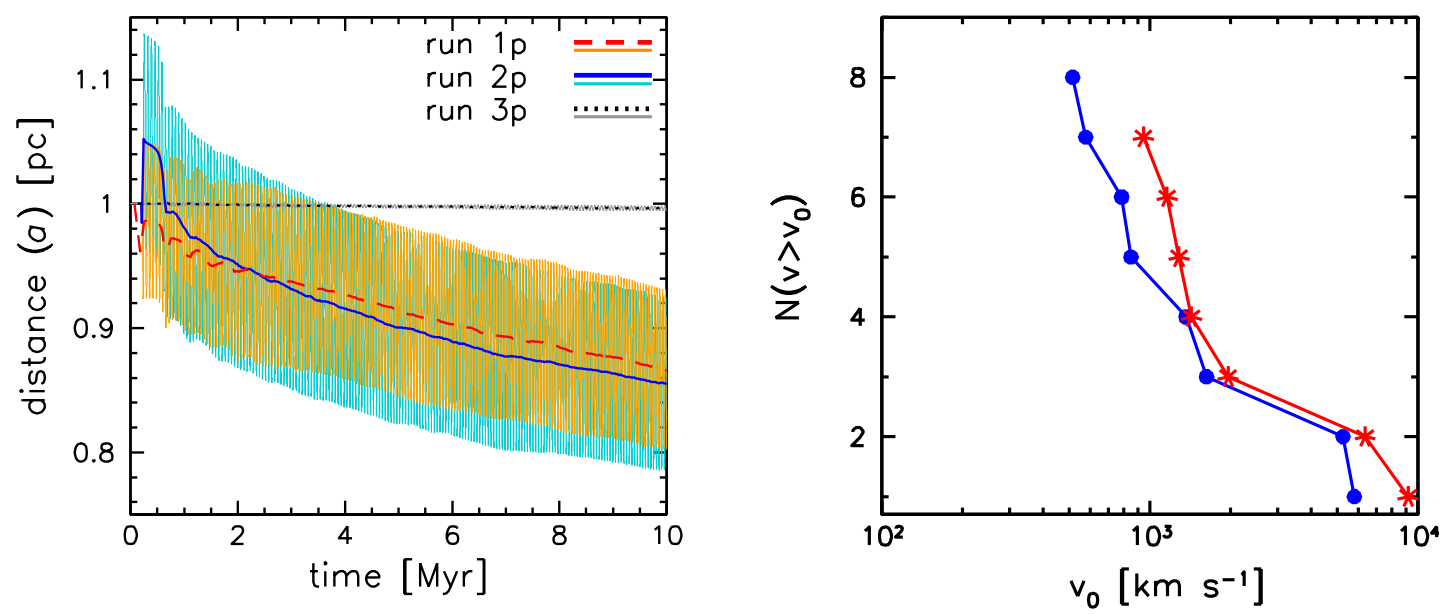

Figure 2: Left-hand panel: time evolution of the BBH separation and semi-major. Red dashed, blue solid and black dotted thick lines show the evolution of the BBH semimajor axis in runs $1 \mathrm{p}, 2 \mathrm{p}, 3 \mathrm{p}$, respectively. The solid thin orange, light blue and grey lines show the time evolution of the BBH separation in the same runs. Right-hand panel: number of unbound stars $N$ whose velocity $v$ is greater than a threshold velocity $v_{0}$, as a function of $v_{0}$ for run $1 \mathrm{p}$ (red line, asterisks) ad run $2 \mathrm{p}$ (blue line, circles). Note that the velocities shown here are computed from a snapshot at $t=10 \mathrm{Myr}$.

\subsection{SCs on non-zero angular momentum orbit}

When some angular momentum is added to the infalling SC (i.e. in run $3 p$, with eccentricity 0.75), the $\mathrm{BBH}$ response to the $\mathrm{SC}$ infall is significantly weaker. In particular, the $\mathrm{BBH}$ semimajor axis shrinks by less than 0.5 per cent in $10 \mathrm{Myr}$ (Fig. 2, left-hand panel), and its hardening rate is always of the order of $s \sim 10^{-4} \mathrm{pc}^{-1} \mathrm{Myr}^{-1}$. Consistently, the BBH eccentricity stays nearly equal to zero throughout the simulation. The difference between run $3 p$ and the other two simulations has to be ascribed to the amount of stars inside the loss cone region: in run 3p, a negligible fraction of stars (less than 0.3 per cent) is found to inhabit the loss cone at all times; in comparison, the fraction of stars on loss cone orbits in runs $1 \mathrm{p}$ and $2 \mathrm{p}$ is always above 25 per cent, and higher than 50 per cent at the first SC periapsis passage.

As a result of the weak interaction, the SC stars settle on a three-lobed discy structure around the $\mathrm{BBH}$ (Fig. 1, bottom right-hand panel), whose external radius is $R \lesssim 20 \mathrm{pc}$ and whose thickness is $\sim 0.1 R$.

\subsection{Hyper-velocity stars}

During the interaction, most stars remain bound to the combined potential of the $\mathrm{BBH}$ and the galaxy throughout the $10 \mathrm{Myr}$ of evolution. This happens because the $\mathrm{BBH}$ is still marginally soft, thus each star is expected to undergo multiple scatterings before being finally ejected from the galaxy. However, a handful of stars manages to escape the combined potential of the SMBHs and the galaxy (7 and 8 stars, respectively) in runs $1 \mathrm{p}$ and $2 \mathrm{p}$. The number of unbound stars whose velocity is greater than a given velocity $v_{0}$ as a function of $v_{0}$ is shown in Fig. 2 (right-hand panel). The escape velocities at the moment of the ejection are always above $1,000 \mathrm{~km} \mathrm{~s}^{-1}$, and can even reach $10,000 \mathrm{~km} \mathrm{~s}^{-1}$; such escaping stars can be classified as genuine hyper-velocity stars. 


\section{Discussion and conclusion}

In the present study, we studied the interaction between a $2 \times 10^{6} \mathrm{M}_{\odot} \mathrm{BBH}$ and an SC weighting roughly $1 / 20$ of the BBH mass. We found that the interaction is rather weak if the SC initial orbit is non-radial, as no significant $\mathrm{BBH}$ hardening results from the SC infall. On the other hand, if the $\mathrm{SC}$ radially approaches the $\mathrm{BBH}$, the binary semimajor axis shrinks by $\sim 10$ per cent in less than 10 Myr. This result suggests that the interaction of a BBH with a $\sim 10$ times more massive $\mathrm{SC}$ (or equivalently, the radial infall of $\sim 10 \mathrm{SCs}$ as massive as the ones simulated in this study) might bring the $\mathrm{BBH}$ close to the GW emission phase.

One may wonder how often we expect a radial SC infall to happen. Several recent studies [17, 18] show that at least part of the star formation observed in the centre of our Galaxy is consistent with being triggered by the collision between molecular clouds. A SC formed as a result of such collision may have a very low angular momentum [19], thus it is expected to infall towards the centre of its host system.

Finally, our work shows that the radial infall of an SC onto a pc scale $\mathrm{BBH}$ may produce a number of hyper-velocity stars, whose velocities might attain values of the order of $10,000 \mathrm{~km} \mathrm{~s}^{-1}$.

\section{Acknowledgments}

We acknowledge financial support from the Istituto Nazionale di Astrofisica (INAF) through a Cycle 31st PhD grant, from the MERAC Foundation and from the Fondazione Ing. Aldo Gini. We also acknowledge the CINECA Award N. HP10CP8A4R and HP10C8653N for the availability of high performance computing resources and support.

\section{References}

[1] M. C. Begelman, R. D. Blandford, and M. J. Rees. Massive black hole binaries in active galactic nuclei. Nature, 287:307-309, 1980.

[2] K. S. Thorne and V. B. Braginskii. Gravitational-wave bursts from the nuclei of distant galaxies and quasars - Proposal for detection using Doppler tracking of interplanetary spacecraft. ApJ, 204:L1-L6, 1976.

[3] P. Amaro-Seoane, H. Audley, S. Babak, J. Baker, E. Barausse, P. Bender, E. Berti, P. Binetruy, et al. Laser Interferometer Space Antenna. arXiv:1702.00786, 2017.

[4] M. Milosavljević and D. Merritt. Long-Term Evolution of Massive Black Hole Binaries. ApJ, 596:860-878, 2003.

[5] E. Bortolas, A. Gualandris, M. Dotti, M. Spera, and M. Mapelli. Brownian motion of massive black hole binaries and the final parsec problem. MNRAS, 461:1023-1031, 2016.

[6] F. M. Khan, D. Fiacconi, L. Mayer, P. Berczik, and A. Just. Swift Coalescence of Supermassive Black Holes in Cosmological Mergers of Massive Galaxies. ApJ, 828:73, 2016.

[7] A. Gualandris, J. I. Read, W. Dehnen, and E. Bortolas. Collisionless loss-cone refilling: there is no final parsec problem. MNRAS, 464:2301-2310, 2017.

[8] Q. Yu. Evolution of massive binary black holes. MNRAS, 331:935-958, 2002. 
[9] E. Bortolas, A. Gualandris, M. Dotti, and J. I. Read. The influence of massive black hole binaries on the morphology of merger remnants. MNRAS, 477:2310-2325, 2018.

[10] D. B. Sanders, B. T. Soifer, J. H. Elias, B. F. Madore, K. Matthews, G. Neugebauer, and N. Z. Scoville. Ultraluminous infrared galaxies and the origin of quasars. ApJ, 325:74-91, 1988.

[11] E. Bortolas, M. Mapelli, and M. Spera. Star cluster disruption by a massive black hole binary. MNRAS, 474:1054-1064, 2018.

[12] M. Arca Sedda, P. Berczik, R. Capuzzo-Dolcetta, G. Fragione, M. Sobolenko, and R. Spurzem. Supermassive black holes coalescence mediated by massive perturbers: gravitational waves emission and the Milky Way - Andromeda fate. arXiv:1712.05810, 2017.

[13] R. Capuzzo-Dolcetta, M. Spera, and D. Punzo. A fully parallel, high precision, N-body code running on hybrid computing platforms. Journal of Computational Physics, 236:580-593, 2013.

[14] W. Dehnen. A Family of Potential-Density Pairs for Spherical Galaxies and Bulges. MNRAS, 265:250, 1993.

[15] I. R. King. The structure of star clusters. III. Some simple dynamical models. AJ, 71:64, 1966.

[16] P. Kroupa. On the variation of the initial mass function. MNRAS, 322:231-246, 2001.

[17] M. Tsuboi, A. Miyazaki, and K. Uehara. Cloud-cloud collision in the Galactic center $50 \mathrm{~km} \mathrm{~s}^{-1}$ molecular cloud. PASJ, 67:109, 2015.

[18] K. Tanaka. ALMA Images of the Host Cloud of the Intermediate-mass Black Hole Candidate CO-0.40-0.22: No Evidence for Cloud-Black Hole Interaction, but Evidence for a Cloud-Cloud Collision. ArXiv:1804.03661, 2018.

[19] M. Mapelli, T. Hayfield, L. Mayer, and J. Wadsley. In Situ Formation of SgrA* Stars Via Disk Fragmentation: Parent Cloud Properties and Thermodynamics. ApJ, 749:168, 2012. 